\title{
Visualisation of Long-term ECG Signals Applied to Post-Intensive Care Patients
}

\author{
Delaram Jarchi*, Adam Mahdi, Lionel Tarassenko and David. A. Clifton
}

\begin{abstract}
A visualisation method for automatically clustering heart beats in single channel electrocardiography (ECG) signals was developed and applied to a dataset of post-intensive care patients who received long-term continuous monitoring. We first segmented the ECG signal into individual beats using an R-peak detection algorithm. A matrix was constructed by storing the segmented ECG beats in row-wise format. Singular value decomposition (SVD) was applied to remove sparse invalid detected $R$ peaks, thus smoothing the matrix. Treating the matrix of ECG beat values as an image, an edge detection algorithm was applied, resulting in a binary matrix containing traces of heart beats with the contiguous and discontiguous components extracted. We considered each component to be a cluster of heart beats. This method was robust to signal noise by exploiting detected $R$ peaks and ECG raw cycles represented in a matrix format for estimation of heart beats. The algorithm also eliminated the effect of underestimated $R$ peaks in the estimation of heart beats, and minimised the effects of overestimated R peaks using the SVD algorithm. This method allows clusters of beats to be visualized, which may assist clinicians in estimating the components of long-term ECG signals.
\end{abstract}

\section{INTRODUCTION}

The relationship between the autonomic nervous system and cardiovascular mortality has been widely accepted. In particular the link between dangerous arrhythmias and abnormal sympathetic or vagal activity has stimulated the development of various quantitative and data visualisation techniques.

Elecrocardiogram (ECG) is a widely used signal for diagnosis of the cardiovascular diseases [1]. Typically, short segments of ECG are inspected for abnormalities but the collection of long-term ECGs is becoming increasingly common. One reason for this paradigm shift is that certain cardiac events tend to take place irregularly and might not occur at the brief recordings in the clinic. The long-term continuous ECG recordings are becoming common mainly due to the availability of the portable ambulatory ECG monitors such as Holter devices [2]. These monitors are capable of recording signals over the course of the day, as the patient perform normal daily activities [3].

The analysis of the data from long-term (e.g. 24-h) ECG can be challenging. The physicians are usually given a statistical summary of the key events that occurred during the recording such as average, minimum and maximum heart rate. These summaries oversimplify the information about the signal dynamics. For example if a patient presents a

This work was supported by EPSRC grant.

*D. Jarchi is with Engineering Science Department, University of Oxford, OX3 7DQ. delaram.jarchi@eng.ox.ac.uk symptom, the doctor may search through the ECG trace to find potential abnormalities for investigation [4], which can be time-consuming and impractical. There is a need to present long-term ECG signals, that could include 100,000 heart beats, in a concise and clear way without omitting the abnormalities [5], [6].

In this work, we introduce a method for automatic clustering and visualisation of long-term ECG recordings with emphasis on identifying epochs of abnormal heart rate. We demonstrate our method in a dataset of ECG signals from post-intensive care patients. The signals are segmented into single beats, which are subsequently transformed into a heat map [7]. If the signal is noisy and beats are misidentified it simply gets ignored and does not bias the overall result.

Applying singular value decomposition (SVD) method filters out sparsely distributed impulse artefacts, as illustrated below.

The heat maps are an easy way to summarise a large number of heart beats in a simple graph. To increase the clarity of the presentation, we convert the heat map into a binary image. The patient's typical heart rate is represented by a thick line with abnormal epochs shown as additional thinner lines. Together with the heat and binary maps we show the corresponding graphs of heart rate, which demonstrate the number of different heart rates existing in the signal and potentially assess the level of abnormality.

The paper is organised as follow. In Section II, the visualisation method is described. Section III provides the results on ECG signals for five patients following their discharge from intensive care unit. Finally, Section IV concludes the paper by highlighting the advantages of the proposed visualisation method and potential improvements for continuous patient monitoring systems.

\section{Method}

Five patients have been selected from voluntary participants in the Post Intensive Care Risk Alerting and Monitoring (PICRAM) - 2 trial. Four out of the five selected patients had arrhythmia. The ECG waveform data were recorded using the Equivital wearable monitoring system with a sampling frequency of $256 \mathrm{~Hz}$. The selected patient data were collected at the John Radcliffe hospital, Oxford, UK. The data corresponds to admissions between 20132014. The raw ECG signals for selected segments including detected R peaks are shown in Fig. 1

The raw ECG signals can contain baseline wander artefact (signal trend) caused by respiration. The baseline wander of the raw ECG signal was removed by applying the singular 
spectrum analysis (SSA) algorithm [8]. The SSA algorithm is an effective, data driven technique that can be used to separate baseline wander from the main oscillatory cardiac component of the ECG signal. The R peaks were then detected in the extracted periodic signal and were used to segment the ECG into individual beats, which were stored row-by-row as a matrix.

For segmentation, the length of each matrix row was long enough to cover the next heart cycle including its corresponding QRS complex. This matrix included sparse intensities due misplaced R peaks. Therefore, SVD algorithm has been chosen to remove those sparse peaks to retrieve a smoothed matrix.

Derived matrix can be considered as an image where the highest intensities are observed around a vertical trace of $\mathrm{R}$ peaks. This image is subjected to an edge detection algorithm, where it has been converted to a binary image with edges detected around regions of $\mathrm{R}$ peaks. The Roberts edge detection algorithm was applied to the matrix, converting it to a binary image with edges detected around regions of $\mathrm{R}$ peaks. Visually, the edge detection algorithm created vertical lines in the image of the matrix during periods of constant heart rate.

The MATLAB regionprops object detection algorithm (MathWorks Inc.) was then applied to the matrix to find contiguous and discontiguous regions of heart beats in order of dimension, thus presenting heart rate clusters in the signal.

The outputs of this function are in order of dimension. This is an important property to visualise components related to various heart rate clusters in decreasing order of heart rates (increasing number of time samples in horizontal dimension).

While the component clusters could be further separated, we present the clusters together in the same figure for simplicity of visualisation. Since the ECG cycles were segmented from the timing of $\mathrm{R}$ peaks of high amplitudes, a short window of samples (10) at the beginning of each matrix row were ignored. The pseudocode for the proposed visualisation technique is shown in Algorithm 1.

In the next section, the results of applying the proposed technique to five patients in the hospital ward following their discharge from intensive care unit are explained.

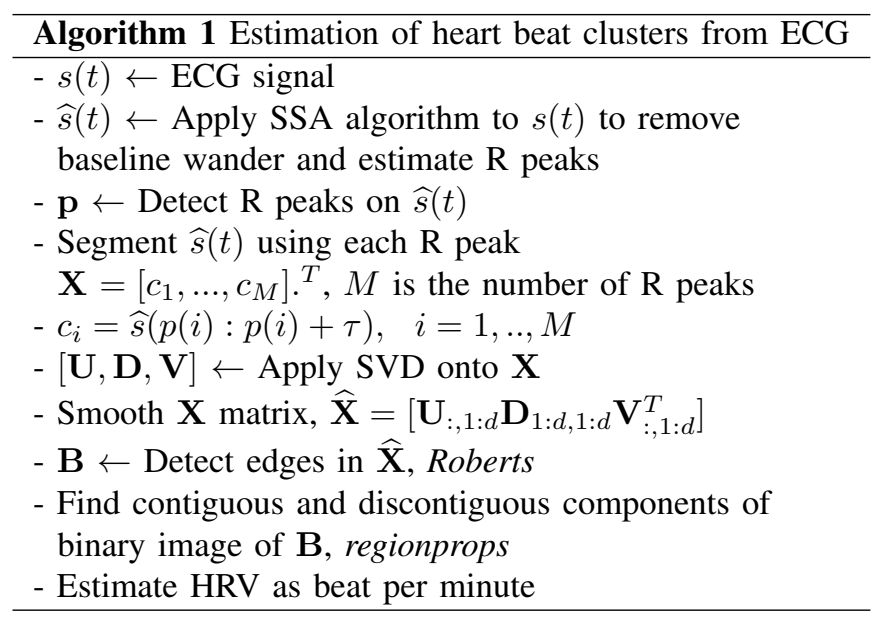

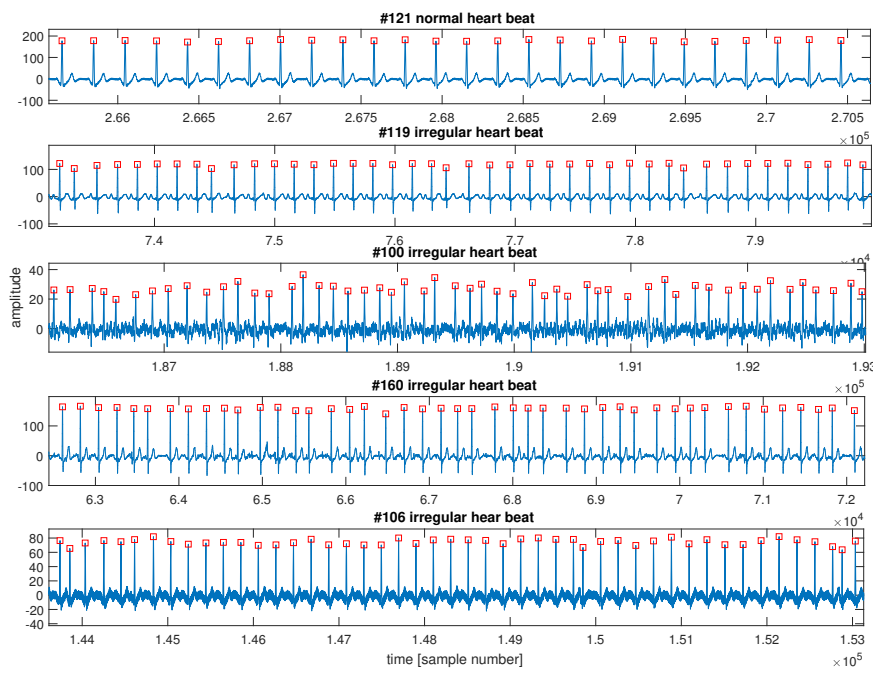

Fig. 1. Selected segments of raw ECG signals for five patients. Only the patient \#121 has shown regular ECG cycles.

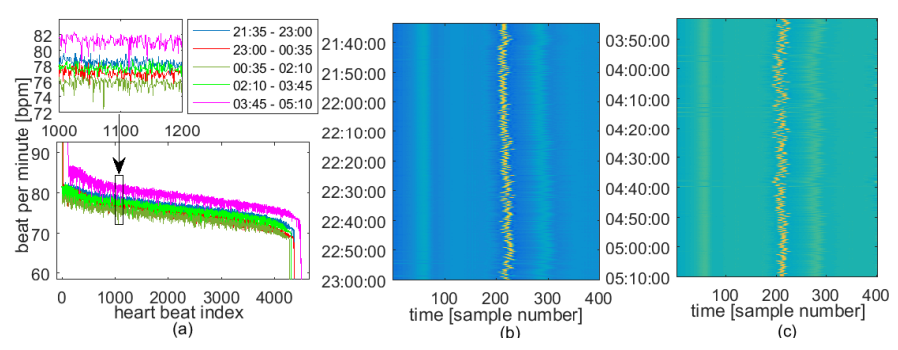

Fig. 2. Heart rate estimates of patient \#121 with normal heart rate. (a) Distribution of Heat rate estimates could be compared over different timewindows. The smoothed matrix corresponding to the first time-window (b) and last time-window (c).

\section{RESULTS}

The proposed method provides a visualisation of heart rates estimated from a long-term ECG signal. The inclusion of five selected patients is to observe differences in the mapped ECG signals and heart rate distributions over time. The selected ECG segments for all five patients are shown in Fig. 1. The results of applying the method to the patient with normal heart rate (\#121) is shown in Fig. 2. The 2D maps of the ECG signals after applying the SVD algorithm are shown for the starting time-window (from 21:35 to 23:00) and last time-window frame (from 03:45 to 05:10) in Fig. 2(b) and Fig. 2(c), respectively. For this patient $\tau$ has been set to 400 . This parameter can be slightly changed for different patients. To cover only one ECG cycle where there are faster heart rates, a smaller value should be considered (e.g. 300 samples). After applying the SVD algorithm, ten eigenvectors with largest eigenvalues are selected to reconstruct the smoothed matrix ( $d=10$ in Algorithm 1).

The estimated heart rates are shown in Fig. 2(a) after applying regionprops to the binary matrix following edge detection. Changes in heart rate estimates over time intervals could been seen from Fig. 2(a). For this patient only one clus- 


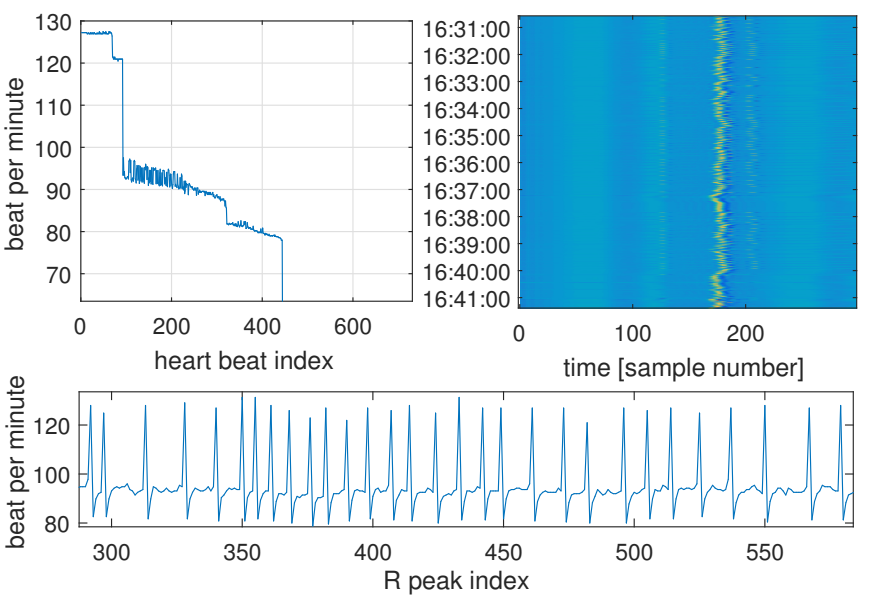

Fig. 3. Heart rate estimates of patient with arrhythmia for patient \#119. Three clusters of heart rate could be observed.

ter of heart beats around valid heart rates can be observed. The other sharp changes in the estimated heart rate relate to invalid estimated heart rates, perhaps due to invalid detected $\mathrm{R}$ peaks or poor quality of ECG signal. Fig. 2(b) provides the resulted smoothed matrix from 21:35 to 23:00. Fig. 2(c) provides estimated smoothed matrix from 03:45 to 05:10. The difference between maximum and minimum heart rate was less than $10 \mathrm{bpm}$ for all time-windows, demonstrating low heart rate variability.

Heat rate estimates for an exemplar patient with irregular heart rates are shown in Fig. 3. In this figure, we can see a trace of high intensities around the main heart rate. Deviations from the main heart rate have been formed as two ranges of the heart rate around $125 \mathrm{bpm}$ and $80 \mathrm{bpm}$. A segmented window of estimated beats per minute from detected $\mathrm{R}$ peaks is shown in the bottom plot of Fig. 3 . This segment clearly shows the main heart rate and two major deviations. In Fig. 4, for 1000 ECG cycles the detected edges on the smoothed matrix including detected components using regionprops have been shown. From this figure, three separate components related to three clusters of heart rates could be observed.

By increasing the length of ECG data segments and cycles, the heart rate estimates seem to converge mainly into two clusters as shown in Fig. 5. (a) for patient \#119. The smoothed matrix of ECG cycles is shown in Fig. 5(b) in the first selected time-window. A small cluster of heart rates around $135 \mathrm{bpm}$ is observed for the first time window (from $16: 30$ to $17: 40$ ). For the last time-window corresponding to the ECG data in the morning (03:00 to 03:55), there are clearly two separate clusters that demonstrate arrhythmia.

In addition, it can be seen that the number of heart rate indices in the cluster related to the main heart rate is decreased for lower heart rate (shown in a circle). This activity can be represented as a separate cluster or deviation from the main heart rate. The difference between minimum and maximum heart rates in the main cluster is about 20bpm for most time-windows. An another example, in Fig. 6, the

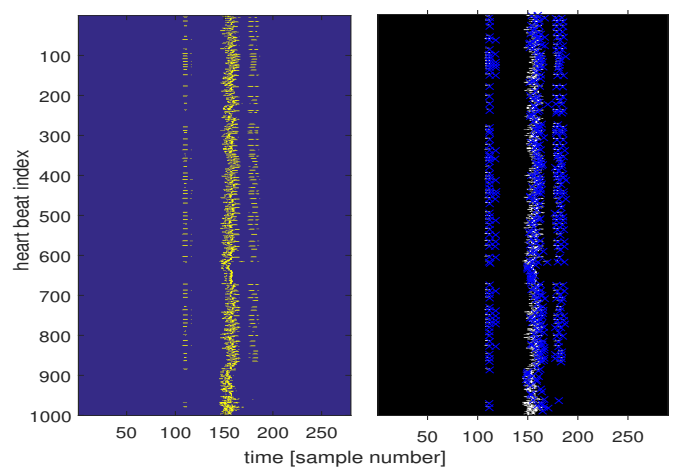

Fig. 4. (a) Detection of edges on the smoothed matrix (b) The results of component identification using regionprops function for patient \#119.

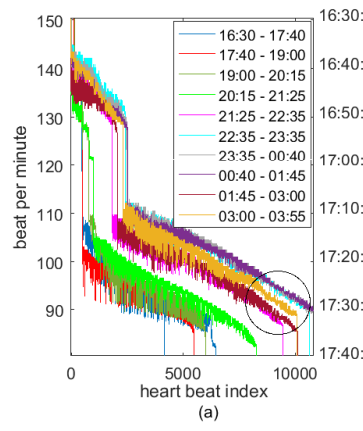

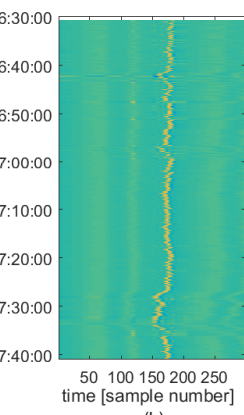

(b)

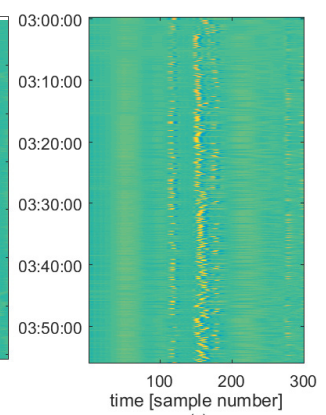

Fig. 5. Visualisation of heart rates from about $4 \mathrm{pm}$ until about $4 \mathrm{am}$ in the morning for patient \#119. Two distinct clusters of heart rate could be observed, although a small cluster (third cluster) can be considered for lower heart rates.

produced image for patient \#160 and estimated R peaks on parts of the signal have been shown. For this patient, 4 clusters of heart rate seem to exist. By adding more signals, four distinct clusters are expected to form.

The results for another patient \#100 with irregular heart rate including a wide range of heart rate variations have been shown in Fig. 8. For this patient the 2D map of the ECG signals was noisy due to large variations in the ECG cycles. Only one cluster of the heart rates has been obtained for most time-windows as seen in Fig. 8. The difference between maximum and minimum ranges of heart rate has been observed to be more than $50 \mathrm{pbm}$ for most time-windows.

To show the effectiveness of the method for handling the low quality ECG signals, patient \#106 has been selected. In Fig. 9, the segmented ECG signal with detected R peaks are shown. This figure shows two episodes where the signal quality of the ECG signal drops. The presented method aims to avoid the effects of invalid detected $\mathrm{R}$ peaks on the estimation of heart rate.

The created binary matrix of ECG cycles is shown in Fig. 10(a). The resulted estimated heart rate is shown in Fig. 10(b). There is a continuous variation in the estimated heart rates which relate to invalid values. Then, a small cluster of heart rates are formed around 70bpm related to the main heart rate. This example shows that there is 

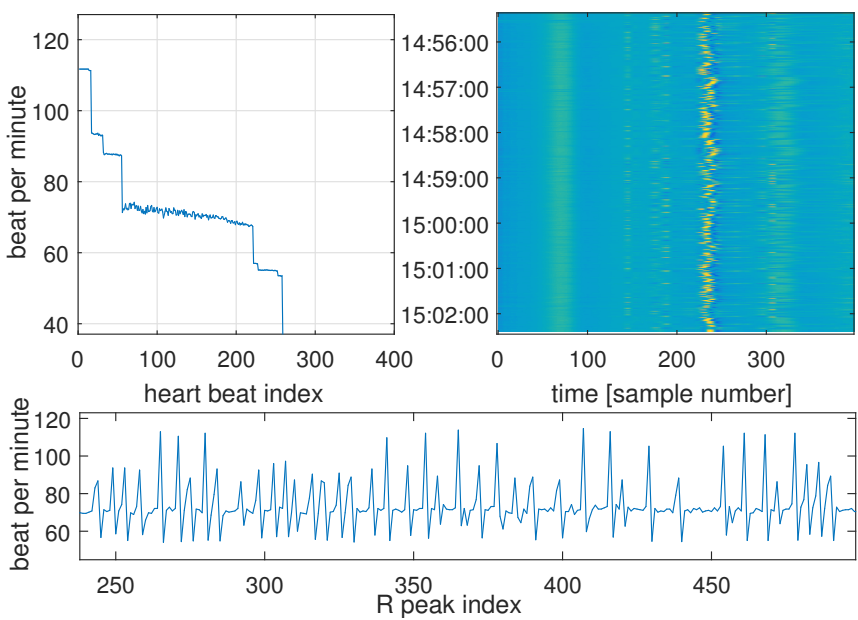

Fig. 6. Heart rate estimates for patient \#160 with arrhythmia. Four clusters of heart rate could be observed.

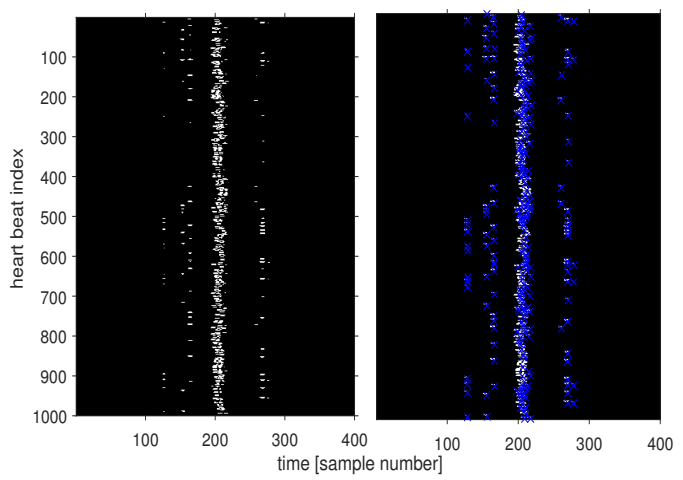

Fig. 7. (a) Detection of edges on the smoothed matrix (b) The results of component identification using regionprops function for patient \#160.

a potential to separate low quality segments of the ECG signals not affecting the actual heart rate estimates. More improvements on quantification of various heart rate clusters can be performed in future studies.

\section{Discussion}

The proposed visualisation technique aims to provide useful information associated with long-term ECG recordings, including the ranges of maximum and minimum heart beats within main clusters. The cluster with maximum number of heart beats could be mainly related to the average heart rate. The other clusters with a lesser number of heart beats will be related to arrhythmia or invalid heart beats, which may be produced due to poor ECG signal quality.

This method combines temporal $\mathrm{R}$ peak information with segmented raw heart cycles and post-processing of matrices to have a reliable estimation of heart beats. The advantage of the method is the insensitivity of the algorithm to underestimated $\mathrm{R}$ peaks.

Although information may be lost due to mis-detected $\mathrm{R}$ peak (underestimation problem), an invalid low heart rate will not be produced to affect any statical measures required for quantification of heart rate variability, which can happen
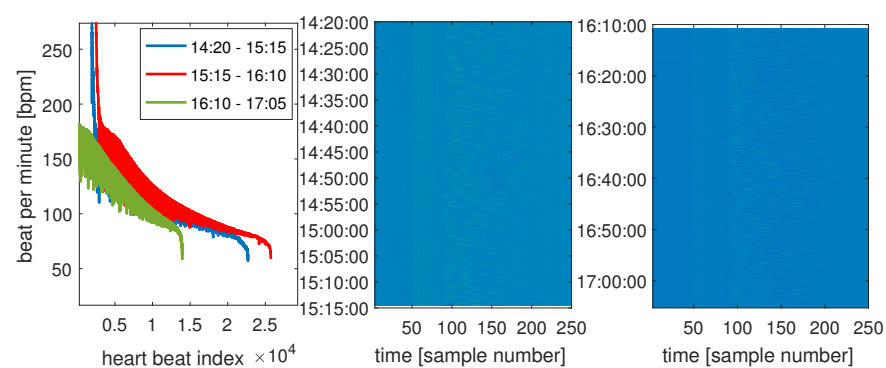

Fig. 8. Heart rate estimates of a patient with irregular heart rate for patient \#100 shown for about 2.5 hours. The resulted matrix and smoothed version do not show a narrow trace of high intensities related to heart rate. The heart rate ranges are from about $80 \mathrm{bpm}$ to $180 \mathrm{bpm}$.

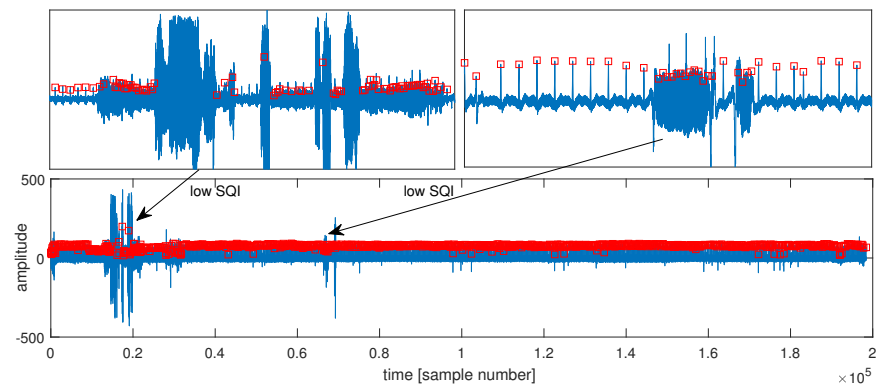

Fig. 9. Top plot: Two parts of the signal where the signal quality drops. Bottom plot: The raw ECG signals and detected R peaks for patient \#106.

when estimating heart rate based on only RR intervals. The effects of sparse overestimated $R$ peaks will be minimised by applying the SVD algorithm to smooth the matrix of the heart cycles. In addition, invalid peaks detected during periods of low signal quality appear as separate components during clustering, which could be investigated in future studies to derive novel signal quality indices.

Visualisation of long-term ECG and derived statistics may assist clinicians to monitor patients' conditions throughout their stay in the hospital to detect arrhythmia and critical conditions such as patient deteriorations. A comprehensive analysis of ECG signals for large number of patients including various types of arrhythmia can be performed in future studies.

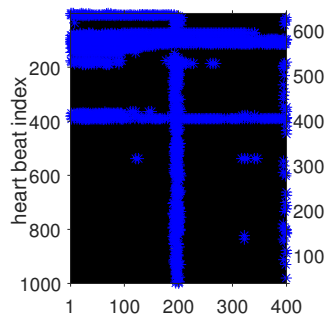

(a)

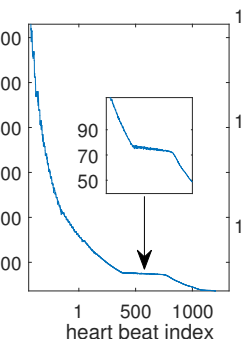

(b)

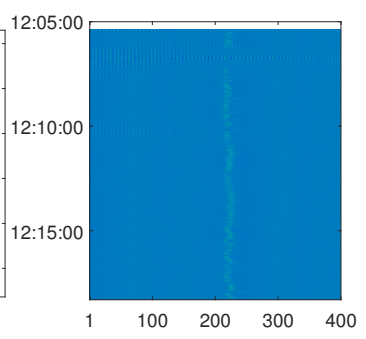

(c)
Fig. 10. Estimated binary matrix for patient \#106 including two episodes of very low quality ECG signals. The estimated beats per minute is shown in the middle plot still shows a constant heart rate around $80 \mathrm{bpm}$ 


\section{REFERENCES}

[1] G. Clifford, C. Liu, B. Moody, L.H. Lehman, I. Silva, Q. Li, A. Johnson, Roger G. Mark. AF Classification from a Short Single Lead ECG Recording: the PhysioNet Computing in Cardiology Challenge 2017. Computing in Cardiology (Rennes: IEEE), Vol 44, 2017 (In Press).

[2] P Ritter "Holter in monitoring of cardiac pacing". Prog Cardiovasc Dis 56: 211-223, 2013.

[3] S.Z. Rosero, V. Kutyifa , B. Olshansky, W. Zareba "Ambulatory ECG monitoring in atrial fibrillation management." Prog Cardiovasc Dis 56: 143-152, 2013.

[4] "Heart rate variability: standards of measurement, physiological interpretation and clinical use. Task Force of the European Society of Cardiology and the North American Society of Pacing and Electrophysiology", European Heart Journal vol 17, 354381, 1996.

[5] A. Page, T. Soyata, JP Couderc, M. Aktas, "An Open Source ECG Clock Generator for Visualization of Long-Term Cardiac Monitoring Data". IEEE Access vol. 3, 2704-2714, 2015.

[6] G.D. Clifford, F. Azuaje, P.E. McSharry "Advanced methods and tools for ECG data analysis", Cambridge, Massachusetts, 2006.

[7] D. Li, F. Tian, S Rengifo1, G. Xu1, M.M. Wang and J. Borjigin, "Electrocardiomatrix: A new method for beat-by-beat visualization and inspection of cardiac signals". J Integr Cardiol, vol 1 124-128, 2015.

[8] D. Jarchi, A. J. Casson, "Towards Photoplethysmography-Based Estimation of Instantaneous Heart Rate During Physical Activity" in IEEE Transactions on Biomedical Engineering, vol. 64, no. 9, 2017, pp. 2042-2053. 